\title{
Relevância da leitura da bula de medicamentos
}

\author{
Relevance of the Reading of the Medicine Insert
}

\author{
Luzia de Castro Macedo, Lidiane Andressa Cavalcante Uhlmann ${ }^{\circ}$ \\ Faculdade de Palmas, Palmas, Tocantins, Brasil. *Autor para correspondência. E-mail: Luzziamacedo665@gmail.com
}

\begin{abstract}
Resumo: Introdução: As informações dos medicamentos são disponibilizadas aos usuários através das bulas, acompanhado de informações que orientam o seu uso adequado. Revisão: A metodologia de elaboração do estudo se deu através de pesquisa bibliográfica de natureza exploratória, que tem como base o levantamento de dados que possam descrever a importância da bula de medicamentos, e seu uso e compreensão entre os usuários através da leitura. Discussão: Segundo diversos autores estudados, muitos usuários não leem as bulas dos medicamentos que consomem, ficando dependente apenas das orientações da forma de uso feitas pelos profissionais de saúde. De acordo com as determinações da ANVISA os usuários precisam ter informações quanto aos medicamentos, e, nesse caso, a bula além de oferecer tais informações, traz especificações legais que garantem este acesso aos pacientes, orientando de forma clara e técnica através de próprias embalagens. Considerações finais: Dessa forma, conclui - se pela necessidade do paciente ler a bula de medicamento, pois facilita a sua automedicação, ajudando no bem estar e melhoria da qualidade de vida.
\end{abstract}

Palavras-chave: medicamentos, leitura, bula, legislação de medicamentos.

\begin{abstract}
Introduction: Information on medications is made available to users through the package inserts, accompanied by information that guides their proper use. Review: The methodology for preparing the study took place through bibliographic research of an exploratory nature, which is based on the collection of data that can describe the importance of the package leaflet, and its use and understanding among users through reading. Discussion: According to several authors studied, many users do not read the package inserts of the drugs they consume, being dependent only on the guidelines for the form of use made by health professionals. According to ANVISA's requirements, users need to have information regarding medications, and, in this case, the package insert, in addition to providing such information, brings legal specifications that guarantee this access to patients, providing clear and technical guidance through the packaging itself. Final considerations: In this way, it is concluded that the patient needs to read the medicine leaflet, as it facilitates self - medication, helping in well - being and improving the quality of life.
\end{abstract}

Keywords: medication, reading, package insert, medication legislation.

\section{Introdução}

A bula de medicamentos é um importante documento relativo à utilização e manutenção de medicamentos, tendo em vista que ela apresenta informações específicas sobre os cuidados e precauções, quanto ao uso dos fármacos e sua composição química (Fujita et. al., 2014).

No Brasil, todos os conteúdos e informações contidas nas bulas de medicamentos são regulamentados pela Agência Nacional de Vigilância Sanitária (ANVISA), que é o órgão institucional do Ministério da Saúde - MS, que tem por finalidade a proteção da saúde da sociedade, através do controle sanitário e dos processos de desenvolvimento de insumos e tecnologias relacionadas a ela (Cunha et al., 2018).

De acordo com a ANVISA, a bula é um documento devidamente regularizado, que atua no direcionamento de informações técnica - científicas de forma orientadora, tanto a profissionais da saúde quanto aos pacientes e usuários, no uso racional de medicamentos. Objetivando a sua garantia e regular utilização e eficácia do conteúdo dos medicamentos, a ANVISA estabelece diretrizes regulamentares para as bulas, como o caso da Resolução - RDC no 140/2003 (ANVISA, 2009).

Com as determinações da ANVISA, torna-se evidente que todos os usuários precisam ter informações quanto aos medicamentos, e, nesse caso, a bula além de oferecer tais informações, traz especificações legais que garantem este acesso aos pacientes, orientando de forma clara e técnica através de próprias embalagens (Nascimento, 2018). 
Sendo o medicamento um produto de consumo, o indivíduo que irá adquiri-lo precisa ter acesso às todas as informações necessárias quanto à forma adequada para esse consumo. Com isso, o medicamento sendo algo que está diretamente relacionado à saúde do indivíduo, está acompanhado da bula para finalidades específicas como aplicação, segurança, armazenamento, riscos e outros (Fujita et al., 2014).

Para Sousa et al. (2014), embora a bula seja um importante documento de orientação para o uso de fármacos, é possível avaliar através de pesquisas que sua aplicação tem sido pouco discutida, comprometendo a utilização, seja por causa da complexidade das informações que, necessariamente precisam estar nela, especificando a composição e efeitos dos medicamentos, e isso se torna um dos motivos de muitos usuários deixem de considerar a sua importância através da leitura.

Diante dessa situação, questiona-se, contudo, quais as regulações já realizadas na bula de medicamentos, e os principais motivos que levam os usuários não darem a devida atenção a essa principal fonte de informações do medicamento.

O presente estudo tem por objetivo descrever a importância da bula de medicamentos, observando suas manutenções legais vigentes, para que os usuários possam realizar uma adequada leitura e interpretação das informações nela contidas. Como objetivos específicos, buscou-se: conhecer a legislação vigente sobre as bulas de medicamentos, identificar a relação existente entre as bulas de medicamentos e seus usuários quanto ao acesso, e verificar os problemas causados pela incompreensão da bula de medicamentos.

\section{Revisão}

O presente estudo trata-se de uma pesquisa bibliográfica de natureza exploratória, que tem como base o levantamento de dados que possam descrever a importância da bula de medicamentos, e seu uso e compreensão entre os usuários através da leitura.

Para se chegar ao resultado desejado, foi feito uma pesquisa bibliográfica, exploratória, com no intuito de obter estudos científicos relacionados ao tema, através das plataformas e banco de dados: ScIELO (ScientificElectronic Library/ Biblioteca Eletrônica Científica Online) LILACS (Literatura Latino-americana e do Caribe em Ciências da Saúde), Medline (Medical LiteratureAnalysisandRetrievel System Online/ Sistema Online de Busca e Análise de Literatura Médica) e o Google Acadêmico.

Dessa forma, os argumentos e os fatos que sustentaram este estudo foram recuperados a partir das palavras-chave: medicamentos, leitura, bula, legislação de medicamentos.

Foi considerado ainda o período entre 2000 a 2020, buscando as informações e dados das últimas duas décadas sobre o tema. Para seleção dos artigos, e composição da revisão da literatura, foram considerados alguns critérios de inclusão, tais como os que abordavam especificamente o tema "bula de medicamentos", no idioma em português. Os critérios de exclusão foram os artigos fora da temática abordada, em outros idiomas, os que não mencionavam data ou autoria do artigo.

\section{Discussão}

Considerando as pesquisas bibliográficas realizadas, são apresentadas a seguir as principais referências deste trabalho, e realizadas as discussões sobre as informações presentes na bula de medicamentos, a legislação vigente, os usuários e a importância da leitura e interpretação de informações nela contidas.

\section{A bula de medicamentos}

De acordo com o dicionário Micha Ellis a bula de medicamento possui a significação: a bula tem origem no latim bulla (bola) [...] Bu.la. sf (latbulla) Impresso que acompanha um medicamento e que contém as indicações necessárias para o respectivo uso."

A bula é o registro impresso das informações relativas aos medicamentos, que acompanha as suas embalagens, prestando auxílio quanto ao uso do fármaco, orientações da composição, fabricantes, os efeitos, as contraindicações, forma de descarte e etc., estando, portanto, destinada a todos os usuários, sejam eles pacientes ou profissionais, e sendo a principal fonte de informações acerca do uso correto de medicamentos (Gonçalves et al., 2013).

A bula de medicamentos é definida pela Agência Nacional de Vigilância Sanitária (ANVISA) como “documento legal sanitário que contém informações técnico-científicas e orientadoras sobre os medicamentos para seu uso racional" (ANVISA, 2009). 
De acordo com Paula et al. (2009) o uso de medicamentos, entre os produtos para consumo, está entre os mais de maior risco para que pessoas consumam de forma irracional, comprometendo à saúde. Por esse motivo a bula é um documento indispensável. No entanto, mesmo quando os medicamentos estão acompanhados de orientações em rótulos do produto e nas bulas, ainda assim não é dada a devida atenção às suas recomendações de uso.

A priori, as informações de remédios e produtos de saúde serviam apenas para serem usados para fins de marketing e não havia um controle por parte da vigilância sanitária sobre o conteúdo das embalagens, dos rótulos, e bulas. Contudo, a partir das regulamentações da Agência Nacional de Vigilância Sanitária ANVISA, as indústrias, fabricantes, e demais envolvidos passaram a desenvolver e fornecer de forma supervisionada todos os dados sobre os medicamentos, como por exemplo, para qual o uso indicado e efeitos diversos (Caldeira et al., 2008).

\section{Análise da legislação da bula de medicamentos}

Atualmente, as bulas de medicamentos são desenvolvidas pelas indústrias farmacêuticas e controladas pela ANVISA, sendo sua dispensação obrigatória, estando garantida por lei (Cunha et al., 2018).

Cunha et al. (2018) explica que no Brasil, entre os anos de 1946 até 2009 foram criados vários dispositivos legais, que posteriormente, foram sendo atualizado pela ANVISA, com várias regulamentações definido a forma de apresentação e uso da bula de medicamentos.

Segundo com informações coletadas por Fujita et al. (2014) a instituição da regulação da bula de medicamento foi iniciada em janeiro de 1946 pelo Ministério da Saúde, aprovando o Regulamento da Indústria Farmacêutica no Brasil, através da edição do Decreto $n^{\circ}$ 20.397/46, formalizando assim os textos que compõem a bula.

Posteriormente, no ano de 1959 a publicação da Portaria no 49 agregava ao Decreto $n^{\circ}$ 20.391/46, as orientações e modelos de rótulos a serem aplicadas na bula de medicamentos, sendo este o primeiro regulamento específico para as bulas.

Já no ano de 1984, com a Portaria $n^{\circ}$ 65, aprovada pela Secretaria Nacional de Vigilância Sanitária (SNFMF), ficou este órgão responsável sobre as informações contidas nas bulas de medicamento, o qual começou por em prática um roteiro específico para os conteúdos das bulas (Fujita et al., 2014).

Contudo, conforme Fernandes (2013) no ano de 1997, com uma nova Portaria $n^{\circ} 110$, a Secretaria de Vigilância Sanitária retificou as informações da sua norma anterior, considerando a estrutura já existente, porém, ajustando outras informações técnicas, como àquelas direcionadas ao paciente, ajustando a sua escrita para uma forma mais fácil de leitura.

Mas, de acordo com Nascimento (2018) o processo regulatório das bulas de medicamento somente ocorreu de forma mais específica em 1999, ano do surgimento da ANVISA, autarquia trabalhando de forma independente, assumindo a responsabilidade com os procedimentos de avaliação e aprovação das bulas elaboradas pela indústria farmacêutica antes de chegarem aos estabelecimentos farmacêuticos. Dessa forma, a ANVISA passou a analisar e aprovar a bula do medicamento.

Conforme explica Nascimento (2018) a ANVISA, órgão vinculado ao Ministério da Saúde do Governo Federal é o instrumento regulador dos textos de bulas, assim como todos os rótulos de medicamentos, atuando na garantia da qualidade de produtos e serviços de saúde, e de acordo com a ANVISA, a bula é o registro legal sanitário de acesso às informações e orientações sobre os medicamentos.

Para Rigotto et al. (2016) as RDC no 140/03 e 47/09 foram muito importantes na constituição de texto com frases de alerta nos textos das bulas no intuito de esclarecer os riscos e perigos do uso de medicamentos, e os casos de suspensão de tratamento ou outros procedimentos de uso sem o conhecimento médico.

A ANVISA publicou a Resolução RDC $n^{\circ} 47 / 09$, em substituição a Resolução RDC $n^{\circ}$ 140/03 estabelecendo novas regras para elaboração, harmonização, atualização, publicação e disponibilização de bulas de medicamentos para paciente e para profissionais de saúde.

Conforme explicam Santos et al. (2019) especificamente em 2009, a ANVISA por meio da Resolução da Diretoria Colegiada, $\mathrm{RDC} \mathrm{n}^{\circ} 47 / 09$, apresentou várias regras para as bulas de medicamentos, determinando alguns dispositivos importantes e definitivos, como o tamanho da fonte, o acesso às recomendações da bula para os deficientes visuais e critérios que especificam o correto uso dos remédios.

Contribuindo com essas informações, Pires et al. (2015) reforça que através da ANVISA, as legislações específicas para bulas passaram a serem revisadas, formando uma nova estrutura de regulamentação, atualizando a Resolução RDC $n^{\circ}$ 140/03, como por exemplo, a formatação do tamanho mínimo exigido para 
as letras dos textos de bula, passando de 01 milímetro para 1,5 milímetros; a integração de informações sobre os riscos para mulheres grávidas; adequação quanto à linguagem das bulas, tornando o seu conteúdo numa forma mais fácil de um entendimento.

Outras considerações regimentares a serem consideradas são a da Portaria da Secretaria de Vigilância Sanitária $\mathrm{n}^{\circ} 110$ que regula a obrigatoriedade de se manter dentro das embalagens dos medicamentos a bula com linguagem acessível para os usuários. A bula possui ainda suas versões eletrônicas disponíveis pela ANVISA, o chamado de Bulário Eletrônico, disponível no site www.anvisa.gov.br/bulas, promovendo vantagens e benefícios de informações online, sendo possível sua localização através do próprio nome do medicamento ou pelo nome da organização farmacêutica que a distribui (Lima \& Belato Júnior, 2018).

Assim, conforme explica Fernandes (2013) as regulamentações definidas para a bula de medicamento, servem de instrumento para a saúde do usuário, pois mantém informado quanto ao medicamento, contribuindo para o progresso da saúde pública.

\section{Informações presentes na bula}

Conforme regula a ANVISA, no Art. $1^{\circ}$ da RDC 140/03, as bulas devem estar com seus conteúdos padronizados, nos termos técnico-científicos e orientadores sobre os medicamentos para a constituição de um uso racional. Essas informações indicam toda a maneira de como utilizar o medicamento, desde o procedimento que deve ser realizado, aos cuidados a serem tomados antes, durante e após o uso (Cunha et al., 2018).

Geralmente, as informações localizadas nas bulas de medicamentos são encontradas em forma de tópicos, quase sempre organizadas na seguinte sequência: definição do medicamento, fórmula e composição, informações ao paciente, indicações e contraindicações, cuidados e precauções, reações adversas, informações direcionadas a mulheres grávidas e outros dados adicionais.

Segundo Pinto (2013) as informações pertinentes a cada fármaco são de inteira responsabilidade da entidade que produz, fornece, importa, ou que seja responsável pelo seu registro, e todos os medicamentos deve obrigatoriamente estar acompanhado de elementos instrutivos, desde a produção até a forma de uso e descarte.

Gonçalves et al. (2013) explica que antigamente os órgãos sanitários eram muito insuficientes em relação aos conteúdos que uma bula deveria trazer de informações sobre os medicamentos, e por muitos anos a legislação foi omissa de orientações na regulação das informações básicas que hoje devem estar nas bulas, como escrita, clareza, indicações e advertências.

A partir de 2003 a ANVISA começou a trazer instruções sobre a forma de apresentação de conteúdo para as informações técnico-científicas nas bulas, atualizando as regulamentações já existentes. Atualmente, as bulas dos medicamentos estão configuradas de acordo com a RDC 47/09 que traz dentre as suas orientações, especificamente, esclarecimento ao paciente, estruturada na forma de perguntas e respostas, de modo que esclareça as dúvidas dos usuários (Pinto, 2013).

Contudo, segundo pesquisas realizadas por Pires et al. (2015) ele aponta que em muitas embalagens de medicamentos, as bulas estão disponíveis apenas para atender a obrigatoriedade e as exigências da ANVISA, quando na verdade deveria haver um maior cuidado com as orientações nelas rotuladas.

Pinto e Silveira (2014) explica que a bula se divide em duas categorias: bula direcionada aos pacientes, sendo deliberada aos locais que atuam na dispensação, e bula direcionada aos profissionais de saúde, com destino hospitalar. Com isso, a bula destinada ao profissional pode conter informações que não estão disponíveis nas bulas destinadas aos pacientes.

A legislação vigente, aplicada pela ANVISA, obriga que todos os medicamentos fabricados no Brasil, ou ainda que importados, comportem suas informações dentro das embalagens, para a orientação tanto do usuário quanto a do profissional da saúde que irá prescrever. Tais informações são fundamentais e obrigatórias, de acordo com a Portaria $n^{\circ}$ 110/97 da Secretaria de Vigilância Sanitária (Pinto \& Silveira, 2014).

Quanto à estrutura das bulas, definida na Resolução da ANVISA, RDC n ${ }^{\circ} 47$ de 08 de setembro de 2009, é possível destacar que: devem ser apresentadas de forma objetiva, específica e básica nas mesmas informações farmacêuticas e administrativas, esclarecendo detalhes de sua composição, separadamente, por exemplo, quando for comprimido ou xarope (Sousa et al., 2014).

A estrutura deve estar ainda nos parâmetros de diagramação, com fontes padronizadas, como tamanho, uso de caixa alta e em negrito para destacar as perguntas e os itens de bula, alinhamento e justificado do texto, espaço entre linhas, com fonte Times New Roman, sendo 08 o tamanho da fonte nas bulas para os profissionais, e 10 nas bulas para os usuários, e cores brancas para o papel, sendo a impressão de cor preta (Cunha et al., 2018). 
A RDC no 47/2009 esteve atenta também quanto ao uso de medicamentos por pessoas que apresentam algum fator especial de saúde, utilizando o termo "populações especiais", sendo elas: crianças, idosos, lactentes, gestantes, diabéticos, alérgicos a um ou mais componentes do medicamento, cardiopatas, hepatopatas, renais crônicos, portadores de doença celíaca, imunodeprimidos, atletas e outros que necessitam de atenção especial ao utilizar determinado medicamento (Cunha et al., 2018).

Esta resolução determina ainda que na bula destinada aos profissionais de saúde, no tratamento direcionado a "populações especiais" devem estar especificadas as contraindicações, as advertências e as precauções e modo de usar.

Dessa forma, Lima e Belato Júnior (2018) explicam que todas as informações dos medicamentos são disponibilizadas aos usuários através das bulas, com um conteúdo que orienta quanto a sua composição química, utilização adequada, possíveis efeitos colaterais, dosagem, precauções, descartes, e outros dados que buscam promover o uso consciente dos medicamentos.

Portando a bula é a única fonte de informação impressa para o usuário do medicamento, e suas as ilustrações facilitam a compreensão do usuário e as descrições nas bulas devem ser claras aos usuários do medicamento, orientando na indicação adequada e contraindicações (Gonçalves et al., 2013).

\section{Motivos que dificultam a leitura da bula}

Segundo pesquisas realizadas por Santos et al. (2019), através do Sistema Nacional de Informações Tóxico-Farmacológicas - SINITOX, mais da metade dos medicamentos comercializados e consumidos no Brasil são de uso não racional, com uma automedicação sem orientação farmacêutica, e sem a busca de informações nas bulas através da leitura.

De acordo com Pires et al. (2015) as dificuldades de leitura da bula de medicamentos, apontadas pelos pacientes têm entre os principais motivos, ela possuir textos considerados muito complexos que dificultam na hora da interpretação. Sendo que essas dificuldades em se entender o conteúdo da bula, geram muitos problemas, como por exemplo, o paciente sem conhecer os riscos, acaba fazendo o uso indevido ou interrompendo o tratamento.

As pesquisas realizadas por Santos et al. (2019) apontam como maior agravante a dificuldade visual pelo texto pequeno, gerando incompreensão do texto, sendo que em relação ao texto contido na bula, deveria ser de fácil entendimento para qualquer usuário do produto.

Sobre essa situação, Fernandes (2013) reforça que quando a bula possui um texto muito pequeno, a leitura e compreensão se tornam ainda mais complexas, exigindo sempre o suporte de um profissional da área da saúde para ajudar na orientação.

De acordo com Dummer (2016) esclarece que nas bulas estão inseridas informações como indicação do medicamento e contraindicações, contendo ainda outras descrições especificas necessárias para cada usuário. Essas informações descritas são direcionadas a dois tipos de leitores, ao paciente e ao profissional de saúde.

Dummer (2016) aponta ainda que evidenciações de que as informações aos usuários e as Informações técnicas no conteúdo das bulas de medicamentos, embora sejam essenciais, na maior parte não satisfazem as necessidades de compreensão na hora de usá-los.

Embora exista todo um trabalho de fiscalização na aplicação de regras consistentes, muitos medicamentos estão acompanhados de bulas em desacordo com as normas técnicas da ANVISA, e automaticamente, desatualizadas (Fernandes, 2013).

Em pesquisas realizadas por Santos et al. (2019) apontaram críticas à qualidade dos textos das bulas dos medicamentos, tais discordâncias incluem erros de ortografia, termos em outros idiomas sem qualquer necessidade, falhas de tradução, e até mesmo a falta de orientações quanto as contraindicações do medicamento. Tais evidências de erros desestimulam o interesse da leitura da bula pelos usuários.

Outros motivos estão relacionados a termos técnico-científicos, sem uma utilização mais clara, acompanhada de uma apresentação de informações mais complexas não atualizadas, ou sem o uso de sinônimos apropriados para uma fácil compreensão por usuários leigos (Fernandes, 2013).

Diante dessas dificuldades, a ANVISA buscou elaborar um roteiro para as bulas, criando regras que visam resolver essas questões, fazendo com que a leitura e compreensão se tornem mais claras, como por exemplos através de frases curtas nas descrições dos medicamentos, através da Resolução RDC n 47/09 estabelecendo novas regras para elaboração, harmonização e atualização das bulas de medicamentos para paciente e para profissionais de saúde (Fujita et al., 2014). 


\section{A importância da leitura e compreensão da bula de medicamentos}

Para Rigotto et al. (2016) é de extrema importância que o paciente entenda a sua necessidade do de ler da bula de medicamentos, até porque a leitura é essencial no dia a dia da vida de qualquer indivíduo, para obter informações e estar atualizado, e em relação às bulas medicamentosas, elas objetivam tornar clara a informação do remédio, a fim de promover um uso racional.

De acordo com estudos feitos por Pinto e Silveira (2014) mesmo contendo informações extremamente importantes, muitos usuários não lêem as bulas que consomem, ficando dependente apenas das orientações de uso dos medicamentos por profissionais de saúde. E mesmo sabendo que a leitura da bula pelo profissional da saúde é muito importante, tanto para ele que o auxiliará nas explicações ao paciente, quanto ao esclarecimento de dúvidas, a leitura da bula acaba sendo a principal fonte de informação aos usuários.

Pinto (2013) cita que quando em certos momentos os profissionais da saúde não prestam todas as informações de uso dos medicamentos, os pacientes precisam buscar - lá através das bulas, o que não ocorre na grande maioria dos casos. E, deixar de ler a bula ou até mesmo a forma incorreta de leitura pode atrapalhar o processo de eficácia do medicamento no organismo, e chegar a causar efeitos contrários ao desejado, prejudicando a saúde.

Importante lembrar que a bula serve como ferramenta para facilitar a automedicação, pois os medicamentos são relevantes para a melhoria da qualidade de vida, reforçando o trabalho de conscientização e de educação dos pacientes alertando quanto aos riscos da automedicação, e ainda da indispensável prescrição médica (Silva et al., 2006).

Com isso, se torna de extrema importância que o usuário faça uma boa leitura da bula, para que assim consiga interpretar os seus efeitos, a informação da indicação e quanto usar de medicamento. A leitura da bula se torna um meio mais prático, entendendo que o seu conteúdo está acessível para ele ficar bem informado, ajudando no seu bem-estar, como também impedir que o medicamento seja usado de forma incorreta, o que levaria a possíveis danos à saúde (Dummer, 2016).

\section{Considerações finais}

A partir das referências apresentadas, juntamente com a legislação abordada neste estudo, é possível perceber o grande auxílio que a bula de medicamentos presta quanto ao uso do fármaco, através das orientações da composição, fabricantes, os efeitos, as contraindicações, forma de descarte e etc., sendo, essas informações destinadas a todos os usuários, sejam eles pacientes ou profissionais.

As bulas dos medicamentos estão configuradas de acordo com a RDC 47/09 que traz dentre as suas orientações, especificamente, as informações ao paciente, estruturada na forma de perguntas e respostas, de forma que esclareça as dúvidas dos pacientes.

Contudo, os autores pesquisados explicam que, embora a bula seja um importante documento de orientação para o uso de medicamentos, é possível avaliar através de suas pesquisas que sua aplicação tem sido pouco discutida, comprometendo a utilização, seja por causa da complexidade das informações que, necessariamente precisam estar nela.

Em algumas pesquisas, alguns autores apontaram críticas referente à qualidade dos textos das bulas dos medicamentos, tais erros incluem os de ortografia, termos em outros idiomas sem muitas necessidades, erros de tradução, e até mesmo a falta de orientações quanto a contraindicações do medicamento. Com essa análise, tem - se as evidências de que os erros desestimulam o interesse da leitura da bula pelos usuários.

Contudo, as RDC n ${ }^{\circ}$ 140/03 e 47/09 foram muito importantes na constituição de texto que ajudam na leitura das bulas, com frases de alerta no intuito de esclarecer os riscos e perigos do uso de medicamentos, e os casos de suspensão de tratamento ou outros procedimentos de uso sem o conhecimento médico.

Dessa forma, é possível concluir que tais informações na bula de medicamentos são extremamente importantes em relação ao produto, tornando indispensável a sua leitura pelos usuários em geral, e que estes precisam se atentar para as orientações, prevenções e cuidados que constam nela.

\section{Referências}

Agência Nacional de Vigilância Sanitária [ANVISA]. Resolução-RDC $n$. 47 de 08 de setembro de 2009. Disponível em:https://pesquisa.in.gov.br/imprensa/jsp/visualiza/index.jsp?jornal=1\&pagina=31\&data=09/09/2009. 
Balbani, A. P. S., Stelzer, L. B., \& Montovani, J. C. 2006. Excipientes de medicamentos e as informações da bula. Revista Brasileira de Otorrinolaringologia, 72, 400-406.

Caldeira, T. R., Neves, E. R. Z., \& Perini, E. 2008. Evolução histórica das bulas de medicamentos no Brasil. Cadernos de Saúde Pública, 24, 737-743.

Cunha, A. M., Nascimento, G., \& Guedes, G. P. 2018. Uma análise sobre as bulas de medicamentos no Brasil. In Anais do XII Brazilian e-Science Workshop. SBC.

Domingues, P. H. F., Galvão, T. F., Andrade, K. R. C. D., Araújo, P. C., Silva, M. T., \& Pereira, M. G. 2017. Prevalência e fatores associados à automedicação em adultos no Distrito Federal: estudo transversal de base populacional. Epidemiologia e Serviços de Saúde, 26, 319-330.

Dummer, R. F. 2016. Análise de sites brasileiros de bulas de medicamentos sob a ótica da busca de informação. 2016. Disponível: http://hdl.handle.net/10183/147255. Acessado em: 07 abr. 2021.

Fernandes, M. M. 2013. Bula de medicamentos. 2013. Disponível: http://repositorio.faema.edu.br:8000/jspui/handle/123456789/1136. Acessado em: 28 de mar. 2021.

Fujita, P. L., Machado, C. J. S., \& Teixeira, M. D. O. 2014. A bula de medicamentos e a regulação de suas configurações em termos de forma e conteúdo no Brasil 1. Saúde e Sociedade, 23, 277-292.

Gonçalves, S. D. A., Melo, G. D., Tokarski, M. H. L., \& Barbosa-Branco, A. 2002. Bulas de medicamentos como instrumento de informação técnico-científica. Revista de Saúde Pública, 36, 33-39.

Lima, E. A. S., \& Belato Júnior, S. G. 2018. A importância da bula no uso responsável dos Medicamentos. Acessado em: 03 de fev. 2021

Nascimento, A. L. S. 2018. Regulamentação de bulas: cenário atual dos medicamentos antirretrovirais no Brasil. Disponível em: https://www.arca.fiocruz.br/handle/icict/26193. Acesso em: 29 de mar. 2021.

Paula, C. D. S., Costa, C. K., Miguel, M. D., Zanin, S. M., \& Spinillo, C. G. 2009. Análise crítica de bulas sob a perspectiva do usuário de medicamentos. Visão Acadêmica, 10(2).

Pinto, J. M., \& Silveira, J. G. 2014. Bulas de medicamentos comercializados no Brasil: em foco a análise da qualidade da informação. Disponível em: http://hdl.handle.net/123456789/2524. Acessado em: 13 mar. 2021.

Pinto. J. M. 2013. Bulas de medicamentos comercializados no Brasil enquanto fontes de informação: em foco a qualidade da informação nelas contidas após a resolução RDC $n^{\circ}$ 47/2009 da ANVISA. Disponível em: https://doi.org/10.1590/S1413-99362013000400019. Acessado em: 17 mar. 2021.

Pires, C., Vigário, M., \& Cavaco, A. 2015. Legibilidade das bulas dos medicamentos: revisão sistemática. Revista de Saúde Pública, 49, 4.

Rigotto, G. C., Oliveira, R. R., Júnior, A. T. T., \& de Souza, J. M. 2016. A bula de medicamentos: a importância da leitura das bulas. Revista Científica da Faculdade de Educação e Meio Ambiente, 7(1), 16-26.

Santos, D. J. L., Feitosa, E. S. M., Dalcin, M. F. 2019. A importância da bula para o usuário de Medicamentos. Disponível: https://www.mastereditora.com.br/periodico/20190607_201024.pdf. Acessado em: 13 de abr. 2021.

Silva, M., Almeida, A. E. D., Oliveira, A. M., Correia, C. C., Benzatti, F. P., Fernandes, J. T., ... \& Doneida, V. C. 2006. Estudo da bula de medicamentos: uma análise da situação. Revista de Ciências Farmacêuticas Básica e Aplicada, 229-236.

Sousa, J. P. R., Garcia, J. L., \& Junior, A. D. F. G. 2014. O paciente e a bula e suas maiores dificuldades. Revista Eletrônica Faculdade Montes Belos, 7(2).

\section{Minicurrículo}

Luzia de Castro Macedo. Graduando em Farmácia pela Faculdade de Palmas (FAPAL) no ano de 2021.

Lidiane Andressa Cavalcante Uhlmann. Mestre em Biodiversidade, Ecologia e Conservação - PPGBEC UFT (2019) e Licenciada em Ciências Biológicas pela Universidade Federal do Tocantins (2016). Docente na Faculdade de Palmas - FAPAL, desde 2019. 


\section{Sépulstísaúde}

Como citar: Macedo, L.C. \& Uhlmann, L.A.C. 2021.Relevância da leitura da bula de medicamentos. Pubsaúde, 7, a209. DOI: https://dx.doi.org/10.31533/pubsaude7.a209

Recebido: 01 jun. 2021.

Revisado e aceito: 14 jun.2021.

Conflito de interesse: os autores declaram, em relação aos produtos e companhias descritos nesse artigo, não ter interesses associativos, comerciais, de propriedade ou financeiros que representem conflito de interesse.

Licenciamento: Este artigo é publicado na modalidade Acesso Aberto sob a licença Creative Commons Atribuição 4.0 (CC-BY 4.0). 